\title{
Malignant mesenchymoma of ulnar nerve: combined sarcoma of nerve sheath and rhabdomyosarcoma
}

\author{
SAMRUAY SHUANGSHOTI AND VASANT CHONGCHET \\ From the Department of Pathology, Faculty of Medicine, Chulalongkorn University, and \\ Department of Surgery, Vajira Metropolis Hospital, Bangkok, Thailand
}

SUMMARY Malignant mesenchymoma within the right ulnar nerve of an 8 year old boy is described. The patient did not have stigmata of von Recklinghausen's neurofibromatosis. The growing and painful tumour was excised five and a half months after detection, and recurred five months later. Mingling of the nerve sheath sarcoma and rhabdomyosarcoma was noted within the same mass which was separated from the adjacent striated muscles. It is suggested that this mesenchymoma arose from mesenchymal cells or cells of mesenchymal type comprising the peripheral nerve sheath which is derived from ectomesenchyme of the neural crest.

We have already recorded two cases of primary intracranial rhabdomyosarcoma (Shuangshoti et al, 1968; Shuangshoti and Phonprasert, 1976). This tumour comprised only $0.1 \%$ of 1028 intracranial neoplasms reported by Shuangshoti and Panyathanya (1974). These authors have also investigated 1274 tumours of peripheral nerve- $61.5 \%$ neurofibromas, $33.5 \%$ neurilemmomas, $0.1 \%$ combined neurofibromas and neurilemmomas, and $4.9 \%$ nerve sheath sarcomas (so-called malignant Schwannomas, neurofibrosarcomas, or neurogenic sarcomas). They found no instances of these peripheral nerve neoplasms containing elements of striated muscle. Only a subcutaneous neurilemmoma had bone formation within the mass which was presented separately (Shuangshoti and Netsky, 1971a). Subsequently a case was encountered of sarcoma of the ulnar nerve which exhibited features of combined nerve sheath sarcoma and rhabdomyosarcoma, forming a malignant mesenchymoma.

\section{Case report}

About eight months before admission to hospital, an 8 year old Thai boy experienced a sharp pain at the distal end of the right forearm in a fall in which his ipsilateral hand strongly hit the ground. Two and a half months later, a progressively growing lump appeared on the ventrolateral aspect of the distal part of the right forearm. He was hospitalised

Address for reprint requests: Dr Samruay Shuangshoti, Department of Pathology, Chulalongkorn Hospital, Bangkok 5, Thailand.

Accepted 16 December 1978 because of severe pain at the affected part, especially when the mass was palpated.

At the time of admission, the body temperature was $36.8^{\circ} \mathrm{C}$; pulse rate, $85 / \mathrm{min}$; respiratory rate, $18 / \mathrm{min}$; and blood pressure, $110 / 80 \mathrm{mmHg}$. The mass was fixed and stony hard. No stigmata of von Recklinghausen's neurofibromatosis were observed. The haemoglobin concentration was $11.3 \mathrm{~g} / \mathrm{dl}$. A leucocyte count disclosed 13300 cells $/ \mathrm{mm}^{3}$ with $76 \%$ neutrophils, $22 \%$ lymphocytes, and $2 \% \%$ monocytes. Radiography of the right forearm revealed swelling of the soft tissue of the affected portion. The underlying bones were normal.

It was noted during surgical removal of the mass that a portion of the right ulnar nerve underneath the flexor carpi ulnaris muscle was distended by the tumour, about $150 \mathrm{~mm}$ in length. Its diameter ranged from 10 to $20 \mathrm{~mm}$. The surrounding skeletal muscles were easily separated from the entire lesion which was then excised.

The specimen (Vajira Metropolis Hospital, 17-S081) consisted of a firm and encapsulated mass. Its grey-white, partly lobular cut surfaces (Fig. 1A) were interspersed with small necrotic foci. It was fixed in formalin, embedded in paraffin, sectioned, and stained with haematoxylin and eosin ( $\mathrm{H}$ and $\mathrm{E})$, Masson's trichrome, Gomori's reticulin, Mallory's phosphotungstic acid haematoxylin (PTAH), and Luxol-fast blue stains.

Microscopically, bundles of the nerve fibre (Fig. 1B) and divergent types of tumour cells were identified in the lesion. Spindle-shaped cells (Fig. 1C and D) resembling both Schwann cells and fibroblasts were arranged in intertwined bands. 

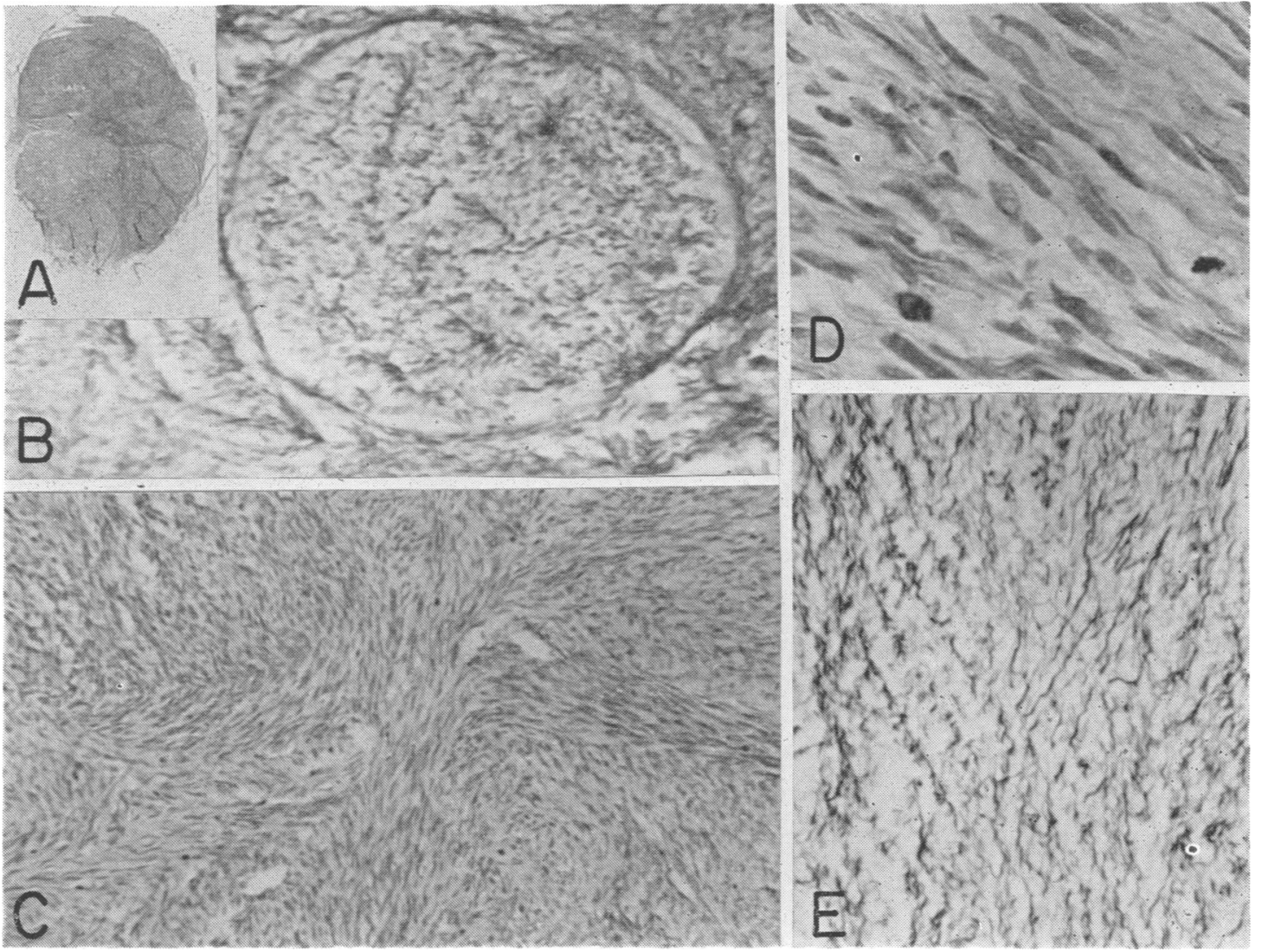

Fig. 1 Features of the tumour: $(A)$ photomicrograph of a transversely cut part of the mass showing its lobular surface ( $H$ and $E, \times 5) ;(B)$ a transversely sectioned bundle of the nerve fibres embedded within the area of the nerve sheath sarcoma $(H$ and $E, \times 100) ;(C)$ intertwined bundles of the spindle-shaped tumour cells in the zone of the nerve sheath sarcoma. There are many mitotic figures but they are not clearly visible at this magnification $(H$ and $E, \times 100) ;(D)$ stream of the spindle-shaped tumour cells and two mitotic figures at greater magnification ( $H$ and $E, \times 400) ;(E)$ dissemination of numerous delicate reticulin fibres in the area of the spindle-shaped neoplastic cells (Gomori's reticulin stain, $\times 100$ ).

Numerous reticulin fibres (Fig. 1E) were scattered among them. The elongated nuclei of the spindle shaped cells were hyperchromatic; mitotic figures, both typical and abnormal, were plentiful. This part of the lesion was diagnosed as nerve sheath sarcoma.

Other types of neoplastic cells were identified. Rounded cells with brightly eosinophilic and either homogeneous or granular cytoplasm in $\mathrm{H}$ and $\mathrm{E}$ preparations (Fig. 2A) were considered to be rhabdomyoblasts. Ballooned cells with vacuolated perikaryon (Fig. 2B) were regarded as spider-web cells (Robbins, 1962). Elongated or strap-shaped cells (Fig. 2C and D), and stellate cells were interpreted as mesenchymal cells (Fig. 2B). Many elongated cells had distinctive cross-striations (Fig. 2C and D) which were best seen with the PTAH and Masson's trichrome stains. The rhab- domyoblasts often were multinucleated--the perikaryon was purplish-red in Masson's trichrome stain, and blue in the PTAH preparation. This was also true for the elongated cells with cross-striations. These various types of the turnour cells were arranged in loose meshes and in bundles as well as disseminated at random among intertwined bands of the spindle-shaped neoplastic cells. Reticulin fibres were few among them, in comparison to those seen in part of the nerve sheath sarcoma. This portion of the neoplasm was diagnosed as rhabdomyosarcoma.

The tumour capsule was often invaded by the neoplastic cells. Clumps of the latter, mainly the spindle-shaped and rounded varieties with abundant mitotic figures, were in a few thin-walled, endothelium-lined spaces within the capsule of the tumour. 

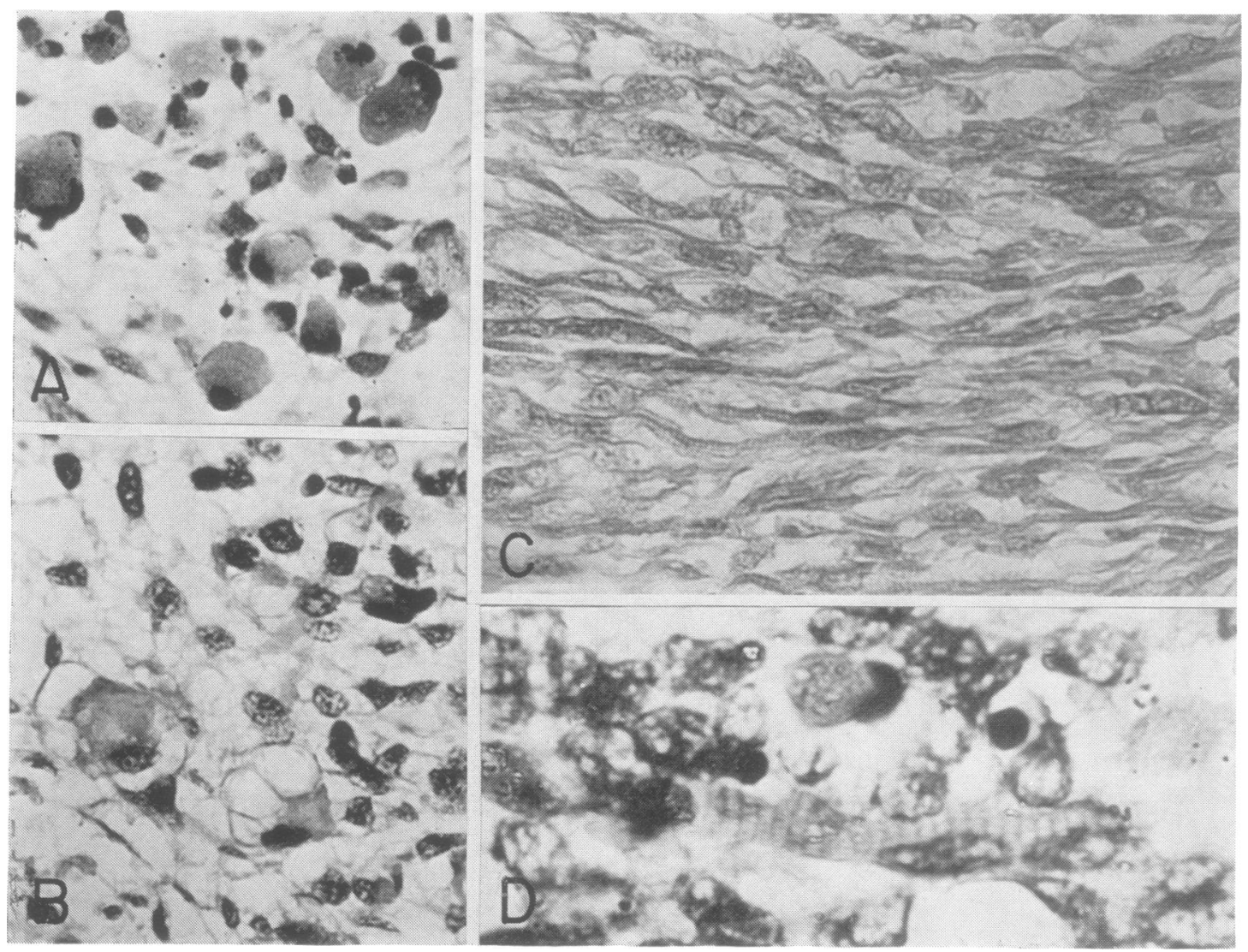

Fig. 2 Rhabdomyosarcomatous part of the neoplasm: $(A)$ rhabdomyoblast $(H$ and $E, \times 400) ;(B)$ spider-web cells and stellate mesenchymal cells $(H$ and $E, \times 400) ;(C)$ elongated cells with cross-striations (Masson's trichrome stain, $\times 400)$; (D) an elongated cell with distinctive cross-striations surrounded by other types of the tumour cells $(H$ and $E, \times 1000)$.

Some spaces contained erythrocytes, and were thus regarded as veins. Others containing no erythrocytes were considered to be lymphatic vessels (Fig. 3). The findings indicated haematogenous and lymphatic spread of the cancer.

Five months after excision, the tumour recurred. The patient's right forearm was amputated at another hospital, and we did not receive information concerning outcome.

The diagnosis of the lesion was malignant mesenchymoma (combined nerve sheath sarcoma and rhabdomyosarcoma) of the right ulnar nerve. Progressive growth of a lump with pain has been observed to be a common presenting feature for malignant mesenchymoma of the peripheral nerve (Woodruff et al, 1973). A traumatic cause has been suggested for a small number of intracranial tumours, such as meningiomas (Zülch, 1965), but we are uncertain whether the mesenchymoma of our case was induced by trauma of the right forearm, two and a half months before the appearance of the lesion. The neoplasm could have preceded trauma. Stigmata of von Recklinghausen's neurofibromatosis have often been noted in cases of malignant mesenchymoma of the peripheral nerve (D'Agostino et al, 1963; White, 1971), but were not seen in our case.

\section{Discussion}

The possibility of collision cancer, such as nerve sheath sarcoma of the ulnar nerve and rhabdomyosarcoma of the adjacent striated muscle, must be considered in our case. Invasion of rhabdomyosarcoma into a sarcoma of the ulnar nerve sheath can create such a combined tumour. However, separation of the surrounding skeletal muscles from the growth within the ulnar nerve as noted during the operation, as well as intimate mingling of the different types of the neoplastic cells within the mass 


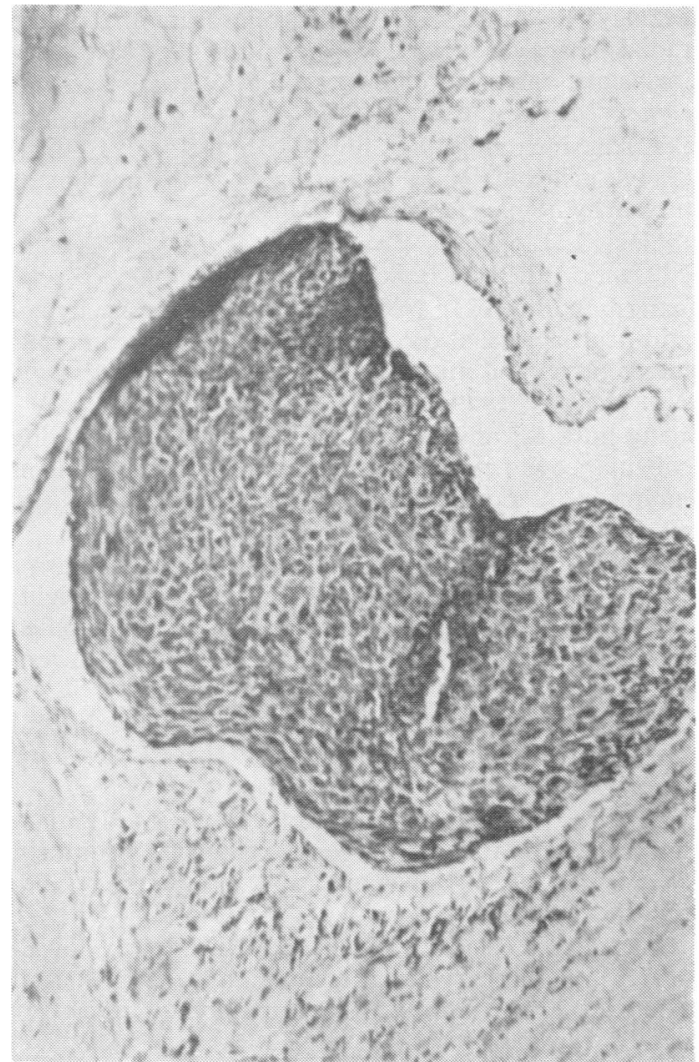

Fig. 3 Clump of tumour cells within the lumen of a lymphatic vessel in the capsule of the neoplasm ( $H$ and $E$, $\times 100$ ).

exclude the possibility of this being a collision tumour. Teratoid or teratomatous nature of the present neoplasm can also be ruled out. These tumours are located in or near the midline of the body. To our knowledge, no cases of teratoid or teratoma of the extremities have been reported.

The mode of occurrence of the present mesenchymoma might be elucidated on the basis of multidirectional differentiation of the mesenchyme or its derivatives. Apart from the myelin sheath, individual axons of the peripheral nerve are also sheathed by neurilemma derived from Schwann cells, and endoneurium of connective tissue origin. Schwann cells are thought to be derived from the neural crest. The tumour in our patient contained numerous spindle-shaped cells which resembled both Schwann cells and fibroblasts. It is thus necessary to determine the nature of the neural crest which might be the origin not only of Schwann cells but of various cells of the connective tissue including fibroblasts.
The neural crest is derived from mingling of mesenchymal cells and developing cells of ectodermal origin at the edge of the neural folds. Hence, it is ectomesenchymal in nature and can differentiate along ectodermal and mesenchymal lines. For instance, cells from the cranial portion of the neural crest migrate to the upper part of the face and form the cranial and some spinal sensory ganglia, autonomic ganglia, sheath cells including Schwann cells, pigmented cells (melanocytes), leptomeninges, membrane bone of the skull, and subcutaneous connective tissue cells including fibroblasts (Johnson, 1966; Weston, 1970). On the other hand, Yntema (1943) demonstrated that sheath cells could be derived from a source other than the neural crest. Bilateral removal of the spinal crest in embryos of Amblystoma punctatum produced deficiency of sheath cells about the proximal parts of the spinal nerve. Distally, the sheath cells were present. With advancing age, they became more numerous and occurred on the nerves nearer the spinal cord (Yntema, 1943). Yntema's experimental findings suggest that the sheath cells can be derived from sources other than the ectomesenchyme of the neural crest. It is reasonable to assume that after removal of the neural crest the sheath cells, including Schwann cells develop from the mesenchyme around the spinal nerves. Yntema's findings, moreover, indirectly suggest that even in the presence of the neural crest the sheath cells would originate from the mesenchymal portion rather than from the ectodermal part of the crest. We thus regard Schwann cells as mesenchymal type cells. An endodermal origin of the sheath cells seems unlikely.

We were unable to decide, using the light microscope, whether the spindle-shaped cells in the tumour of our case were Schwannian or fibroblastic. However, portions of the growth where the spindle-shaped cells were present were clearly sarcoma of these cells which contained numerous reticulin fibres. Thus, the spindle-shaped cells were considered to be mesenchymal in type.

The presence of mesenchymal cells as well as other mesenchymal type celis in this tumour might clarify its mode of occurrence. It has been suggested that multipotential mesenchymal cells and other mesenchymal type cells differentiate into both nerve sheath sarcoma and rhabdomyosarcoma. The mesenchymal cells and mesenchymal type cells may differentiate into fibrous, osseous, chondrous, adipose, muscular, vascular, and reticuloendothelial tissues (Willis, 1948). It is thus not surprising to find heterologous tissues within mesenchymal tumours of peripheral nerve, or in a mesenchymal portion of neoplasms of mixed mesenchymal and neuroepithelial origin of the neuraxis-for example, 
osteoid (White, 1971), bone (Shuangshoti and Netsky, 1971a; Shuangshoti et al. 1971a), cartilage (Shuangshoti and Netsky, 1971b; Shuangshoti et al. 1971a), benign and malignant melanocytes (Bednar, 1957; Fu et al. 1975; Hahn et al. 1976a,b), malignant adipose tissue (Shuangshoti, 1973; Naka et al., 1975), maligant blood vessels (Macaulay, 1978), and malignant striated muscle as in the present instance (Shuangshoti and Netsky, 1971b; Shuangshoti et al., 1971b; White, 1971; Woodruff et al., 1973). It is not surprising either to find neoplasms of mixed mesenchymal and neuroepithelial derivatives outside the neuraxis. For example, Naka et al. (1975) reported a 2 year old girl who had a retroperitoneal tumour and died within six months of admission to hospital, in spite of partial surgical removal, chemotherapy, and radiotherapy. At necropsy, the neoplasm consisting of areas of rhabdomyosarcoma, liposarcoma, undifferentiated mesenchymoma containing cartilage, and ganglioneuroblastoma had metastasised to the right pulmonary hilar lymph nodes. A right sided facial neoplasm of a 6 year old girl described by Karcioglu et al. (1977) was composed of foci of Schwannoma, embryonal rhabdomyosarcoma, clusters of benign melanocytes, and mature and immature ganglioneuroma. The pathogenesis of the tumours in both cases can be clarified by the bidermal nature of the neural crest derivatives where the growths have originated.

\section{References}

Bednar, B. (1957). Storiform neurofibromas of the skin, pigmented and non-pigmented. Cancer, 10, 368-376.

D'Agostino, A. N., Soule, E. H., and Miller, R. H. (1963). Sarcomas of the peripheral nerves and somatic soft tissues associated with multiple neurofibromatosis. Cancer, 16, 1015-1027.

Fu, Y. S., Kaye, G. I., and Lattes, R. (1975). Primary malignant melanocytic tumors of the sympathetic ganglia with ultrastructural study of one. Cancer, 36, 2029-2041.

Hahn, J. F., Netsky, M. G., Butler, A. B., and Sperber, E. E. (1976a). Pigmented ganglioneuroblastoma: relation of melanin and lipofuscin to Schwannomas and other tumors of neural crest origin. Journal of Neuropathology and Experimental Neurology, 35, 393-403.

Hahn, J. F., Sperber, E. E., and Netsky, M. G. (1976b). Melanotic neuroectodermal tumors of the brain and skull. Journal of Neuropathology and Experimental Neurology, 35, 508-519.

Johnson, M. (1966). A radioautographic study of the migration and fate of cranial neural crest cells in the chick embryo. Anatomical Record, 156, 143-155.

Karcioglu, Z., Someren, A., and Mathes, S. J. (1977). Ectomesenchymoma: a malignant tumor of migratory neural crest (ectomesenchyme) remnants showing ganglionic, Schwannian, melanocytic and rhabdomyocytic differentiation. Cancer, 39, 2486-2496.
Macaulay, R. A. A. (1978). Neurofibrosarcoma of the radial nerve in von Recklinghausen's disease with metastatic angiosarcoma. Journal of Neurology, Neurosurgery, and Psychiatry, 41, 474-478.

Naka, A., Matsumoto, S., Shirai, T., and Itoh, T. (1975). Ganglioneuroblastoma associated with malignant mesenchymoma. Cancer, 36, 1050-1056.

Robbins, S. L. (1962). Textbook of Pathology, second edition, p. 1063. W. B. Saunders: Philadelphia.

Shuangshoti, S. (1973). Neoplasm of mixed mesenchymal and neuroepithelial origin: combined liposarcomatous meningioma and mixed gliomas. Journal of Neurology, Neurosurgery, and Psychiatry, 36, 377-382.

Shuangshoti, S., and Netsky, M. G. (1971a). Ossifying subcutaneous neurilemmoma: evidence that neurilemmoma is of mesenchymal origin. Journal of Medical Association of Thailand, 54, 434-444.

Shuangshoti, S., and Netsky, M. G. (1971b). Neoplasms of mixed mesenchymal and neuroepithelial origin: relation to "monstrocellular sarcoma" or "giant-celled glioblastoma". Journal of Neuropathology and Experimental Neurology, 30, 290-309.

Shuangshoti, S., and Panyathanya, R. (1974). Neural neoplasms in Thailand: a study of 2897 cases. Neurology (Minneapolis), 24, 1127-1134.

Shuangshoti, S., and Phonprasert, C. (1976). Primary intracranial rhabdomyosarcoma producing proptosis. Journal of Neurology, Neurosurgery, and Psychiatry, 39, 531-535.

Shuangshoti, S., Netsky, M. G., and Fitz-Hugh, G. H. (1971a). Parapharyngeal meningioma with special reference to cell of origin. Annals of Otology, Rhinology and Laryngology, 80, 464-473.

Shuangshoti, S., Netsky, M. G., and Jane, J. A. (1971b). Neoplasms of mixed mesenchymal and neuroepithelial type: with consideration of the relationship between meningioma and neurilemmoma. Journal of the Neurological Sciences, 14, 277-291.

Shuangshoti, S., Piyaratn, P., and Viriyapanich, P. L. (1968). Primary rhabdomyosarcoma of cerebellum: necropsy report. Cancer, 22, 367-371.

Weston, J. A. (1970). The migration and differentiation of neural crest cells. In Advances in Morphogenesis, vol. 8, pp. 41-144. Edited by H. Abercrombie, J. Brachet, and T. King. Academic Press: New York.

White, H. R. (1971). Survival in malignant Schwannoma. Cancer, 27, 720-729.

Willis, R. A. (1948). Pathology of Tumours, p. 718. Butterworth: London.

Woodruff, J. M., Chernik, N. L., Smith, M. G., Millett, W. B., and Foote, F. W. (1973). Peripheral nerve tumors with rhabdomyosarcomatous differentiation (malignant "Triton" tumours). Cancer, 32, 426-439.

Yntema, C. L. (1943). Deficient efferent innervation of the extremities in Amblystoma. Journal of Experimental Zoology, 94, 319-349.

Zülch, J. K. (1965). Brain Tumors: Their Biology and Pathology, second edition, pp. 51-58. Springer: New York. 